\title{
VIVIR LA CIUDAD: EXPERIENCIAS DE MAESTROS EN EL CONTEXTO URBANO DE POPAYÁN
}

\author{
Alexander Buendía Astudillo* \\ Deibar René Hurtado Herrera** \\ María Andrea Simmonds Tabbert**
}

RESUMEN: Los imaginarios de la ciudad de un grupo de maestros y maestras, analizados desde una perspectiva comunicativa, es el foco central del presente trabajo. Teniendo en cuenta que la ciudad es un espacio comunicativo, esta investigación -desarrollada en el marco de un proceso formativo con docentes- abordó cómo se es visible y cómo se transita en la ciudad. Los relatos biográficos de los maestros y maestras con respecto a la ciudad y la juventud fueron claves para comprender mejor cómo se construyen los imaginarios de ciudad en relación con procesos y medios de comunicación, y cómo éstos tienen relación con su práctica docente.

Palabras-clave: Ciudad; Comunicación; Educación; Maestros; Medios de Comunicación.

LIVING THE CITY:

\section{EXPERIENCES OF TEACHERS IN THE URBAN CONTEXT OF POPAYÁN}

ABSTRACT: The imaginaries of the city of a teacher's group, analyzed from a communicative perspective are the central focus of this paper. Taking into account that the city is a communicative place, this inquiry - carried out in the context of a learning process with teachers - addressed how it is visible and how it travels in the city. The teachers' biographical narratives about the city and the youth were the keys to better understand how the imaginary of the city is made in relation to the processes and media, and how these relate to their teaching practice.

Keywords: City; Communication; Education; Teachers; Mass Media.

\footnotetext{
* Comunicador social-periodista, Magíster en Estudios de la Cultura. Profesor del Departamento de Comunicación Social de la Universidad del Cauca y coordinador del grupo de investigación Estudios culturales y de la comunicación-Ecco. Doctor (c) en Ciencias de la Educación, Rudecolombia-Universidad del Cauca.E-mail: abuendia@unicauca.edu.co

** Doctor en Ciencias Sociales, Niñez y Juventud. Universidad de Manizales-Centro de estudios avanzados en Niñez y Juventud, Educación y Desarrollo Humano/CINDE. Docente de la Universidad del Cauca. Director del grupo de investigación Urdimbre.E-mail: deibarh@unicauca.edu.co, deibarh@yahoo.es

** Magíster en Educación y Desarrollo Humano. Universidad de Manizales-Centro de estudios avanzados en Niñez y Juventud, Educación y Desarrollo Humano/CINDE. Docente de la Universidad del Cauca. Co-fundadora del grupo de investigación Urdimbre.E-mail: masimmonds@unicauca.edu.co
} 


\section{Introducción}

Este texto recoge los resultados de una investigación sobre imaginarios de ciudad y comunicación. El trabajo se realizó con un grupo de maestros (de ambos sexos) en lo que denominamos "visibilidad y transitoriedad en la ciudad". Los docentes son profesores de escuelas y colegios (fundamentalmente públicos) del municipio de Popayán (Colombia). Los centros educativos en los que los maestros ejercen su actividad docente se encuentran ubicados, en su mayoría, en el casco urbano de la ciudad.

Para el ejercicio investigativo, partimos de la premisa que la ciudad es un espacio de y para la comunicación y que esto se da gracias a una serie de dinámicas sociales que están presentes en el contexto urbano. El trabajo con los maestros implicó una primera aproximación conceptual al asunto urbano desde la perspectiva de las dinámicas sociales y de la comunicación. Posteriormente se ha realizado una serie de talleres en los que se utilizaron relatos biográficos de los maestros que escribieron sobre sí mismos.

Este artículo expone cuál fue la aproximación conceptual llevada a cabo en la investigación y el camino metodológico que se recorrió en cada uno de los talleres para abordar las dinámicas urbanas que fueron el eje de la discusión conceptual. Al final se recogen las conclusiones que emergieron del trabajo con los maestros en las diferentes sesiones y talleres.

\section{Referentes conceptuales}

Conceptualmente, se ha trabajado en torno a tres ejes temáticos que se interrelacionan pues son complementarios e interdependientes entre sí; a saber:

a) La ciudad

b) Las dinámicas urbanas

c) La relación -comunicativa- de las dinámicas urbanas y el territorio.

Para abordar estos ejes se plantearon unas preguntas guía:

1. ¿Qué es la ciudad? Aquí se indagó en torno a la ciudad como espacio vital y de sentidos. La indagación apuntó a conocer qué significados se le dan a la ciudad y cómo los sujetos se relacionan entre sí y con el 
espacio urbano. Lo clave era re-conocer la ciudad como territorio por donde circula la comunicación.

2. ¿Cómo comprender/interpretar los sentidos de la transitoriedad y la visibilidad en la ciudad? Se buscaba entender cómo la apropiación de la ciudad pasaba por la apropiación del territorio que se recorre. Asimismo era importante conocer cómo los sujetos urbanos se hacen visibles en la ciudad gracias al uso, consumo y producción de medios (y mensajes) de comunicación, no necesariamente masivos.

Estas preguntas sirvieron de motivación para que se adentrara en la discusión y también para orientar los talleres prácticos que contempló la investigación. En consecuencia, las preguntas pueden asumirse de dos maneras: a) como guía, en tanto determinan el rumbo de las reflexiones teóricas, o b) como provocación, pues a partir de ellas se ha generado un trabajo que lleva a pensar lo práctico, lo vivencial de los conceptos.

\section{La ciudad: escenario comunicacional y territorio de sentidos}

Vista desde la comunicación, la ciudad pasa de simple espacio físico y representación cartográfica, a complejo territorio dotado de sentido. Pero se trata de un sentido que se transforma gracias a quienes habitan la ciudad. Son entonces los habitantes urbanos quienes generan los cambios en la manera en que se vive y percibe un determinado espacio urbano.

Según Carrión (1999) esta relación simbiótica entre ciudad y habitantes se explica - desde la comunicación- a partir de una realidad histórica permanentemente dinámica. La ciudad es foro, por excelencia, también es vitrina, puede ser cárcel pero, sobre todo, es espacio vital. Así, la ciudad termina convertida en un medio de comunicación especial, pero también espacial.

En este escenario destacan una serie de elementos que hay que tener en cuenta para darle seguimiento a la reflexión: hay que asomarse sobre conceptos como lo público y lo privado, el espacio y, como elemento clave, lo urbano; lo cual, según Ulloa (2000: 77) puede definirse como el resultado de la relación dialéctica entre la ciudad que habitamos y la ciudad que nos habita. Lo urbano no es entonces la volumetría física de los edificios, calles o equipamientos; tampoco es la congestión vehicular ni la contaminación ambiental de la ciudad; ni es la suma de la ciudad, con sus 
habitantes y sus problemas. Lo urbano, en este caso, se refiere a la relación entre la espacialidad y los sujetos sociales [...] lo urbano expresa las representaciones individuales y colectivas de la espacialidad o del espacio socialmente construido.

Desde esta perspectiva, es importante que se vea a la ciudad como constituyente de ciudadanos, los cuales adquieren dicha condición no por una cuestión formal o legal, sino por una dinámica social que los transforma pero a la que también tienen la posibilidad de transformar. Vista así, la ciudadanía es un modus vivendi, que se produce gracias a las relaciones de los individuos en un marco social; en dicho marco la comunicación juega un papel determinante que facilita la socialización.

La comunicación en la ciudad es «el conjunto de relaciones sociales y su producto» (WOLLRAD, 1999: 17). Allí, en lo relacional, hay toda una veta de análisis por explorar e interpretar. Justamente la intencionalidad del ejercicio investigativo con los maestros que aquí se expone gira en torno al interés académico por comprender lo urbano desde lo comunicativo, y especialmente aquello que se origina en contextos escolares.

Así las cosas, es importante comprender las relaciones que se dan en la ciudad a partir de las dinámicas urbanas y de procesos de comunicación. Para esto es clave asumir la ciudad como «la construcción social e histórica que, dotada de sentido por el hombre, se vuelve habitable; es el lugar donde nos humanizamos y donde perdemos la humanidad que tenemos. Es un complejo tejido de hibridaciones sociales en el cual sentimos, pensamos, soñamos, creamos, en suma, vivimos» (BUENDÍA, 2006: 42).

\section{Las dinámicas urbanas}

Si bien este concepto amerita un mayor desarrollo teórico, para efectos del trabajo de investigación las dinámicas urbanas se asumieron como aquellos elementos de la vida cotidiana que hacen más fácil que se establezcan relaciones sociales; éstas, en consecuencia, determinan la vida social y urbana y moldean la experiencia vital de la ciudad. A continuación se presentan unas notas para que mejor se comprendan las dinámicas urbanas trabajadas.

\section{a) Notas para pensar la transitoriedad}

(Dime por dónde andas y te diré qué conoces). 
Esta dinámica urbana tiene que ver con los tránsitos y recorridos que ocurren al interior de la ciudad; es decir, tiene que ver con la movilidad pero no se restringe a ella, más bien se trata de cómo se dotan de sentido los espacios que se recorren.

¿Qué circula por la ciudad? ¿Qué flujos se realizan? ¿Quiénes y qué transitan por la ciudad? ¿Cuáles son los recorridos habituales y cuáles son travesías eventuales? Éstas son algunas de las preguntas que nos llevan a indagar acerca de esta dinámica.

De acuerdo con Feixa (1998), un primer paso hacia la apropiación/construcción de territorio es conocerlo, recorrerlo. Los jóvenes, por ejemplo, son básicamente, «gitanos urbanos»; recorren la ciudad por varios motivos: en busca de diversión, lugares de encuentro, esparcimiento, ocio y deporte, por motivos de trabajo, vivienda o amor (BUENDÍA \& HURTADO, 2009). Por eso, quizá, son los sujetos sociales que mejor encarnan y viven la dinámica de la transitoriedad pues son ellos quienes más transitan por la ciudad.

Martín-Barbero (1995: 192), por su parte, señala que «hay un cambio muy fuerte en los modos de relación de la gente joven con el territorio [...] ello tiene lugar porque la gente joven de hoy vive una muy peculiar relación entre su ciudad, su lugar, donde vive, y el mundo». Y eso se debe básicamente a que los jóvenes, con sus prácticas sociales y culturales, crean su propio territorio en el espacio urbano en la medida en que "marcan" los lugares al usarlos o mediante diferentes estrategias de nominación y designación (BUENDÍA \& HURTADO, 2009).

Los jóvenes se apropian, dotan de sentido, resignifican y usan los espacios de la ciudad. Su relación con ellos va desde una perspectiva de territorialidad transitada hasta otra transitable. No hay zonas estáticas, no hay lugares eternos, no hay pausas ni paréntesis urbanos porque la lógica del flujo es más aprehensible y menos condicionante que los puntos geográficos inamovibles. En este contexto es donde se puede comprender mejor la dinámica urbana de la transitoriedad.

Esta dinámica se resume bastante bien en Carles Feixa (1998: 106) cuando plantea que los jóvenes

se han apropiado de determinados espacios libres de la ciudad, y los han humanizado a través del ocio. La juventud es uno de los grupos sociales que establece unas relaciones más intensas con el territorio [...] los jóvenes se han apropiado históricamente de espacios públicos de la ciudad para construir su 
precaria identidad social [...] La acción de los jóvenes sirve para redescubrir territorios urbanos olvidados o marginales, para dotar de nuevos significados determinadas zonas de la ciudad, para humanizar plazas y calles construidas de nuevo [...] Por lo que puede decirse que las culturas juveniles construyen, más allá de la urbe hegemónica que se ve, una verdadera ciudad invisible.

\section{b) Notas para pensar la visibilidad \\ (¿Qué ves, cómo lo ves; cómo ves lo que no ves?)}

Esta dinámica está relacionada con lo visible de y en la ciudad. Asimismo con aquello que propicia dicha visibilidad y aquello que la dificulta. Algunas preguntas que ayudan a develar el asunto de la visibilidad pueden ser: ¿qué o quién se hace visible en la ciudad? ¿Cómo se busca o se logra dicha visibilidad? ¿Por qué y para qué se es visible en la ciudad? Así como aparece la visibilidad, su contraparte, la invisibilidad, también tiene gran relevancia en esta dinámica urbana.

Para este trabajo se asume la visibilidad desde la perspectiva de Pérez Tornero (1998), quien la cataloga como «un valor central de la sociedad consumista», dado que el sentido de la vista y la imagen han sido privilegiados durante todo el siglo XX y el inicio del XXI. Es decir, el ser visible posee una carga valorativa positiva y, por el contrario, el no serlo, se lee, socialmente, como algo negativo.

La invisibilidad, en términos generales, no es en sí un valor sino un desconocimiento, por lo tanto, lo importante es ser visible y hacer visible lo que se es y lo que se hace. No obstante, hay grupos -que pueden catalogarse como marginales- que deben su supervivencia justamente a la invisibilidad, pero esto refuerza la idea de que la visibilidad es sinónimo de existencia. Lo que no se ve, no se conoce y no se sabe que existe.

Los jóvenes y más los grupos de jóvenes se mueven en esta lógica. Y no se trata de un pretendido snobismo sino, más bien, de una búsqueda por el reconocimiento y la validez; en últimas, por la legitimación social.

En este panorama es donde aparece la comunicación asociada a los medios masivos. Estos se convierten en una forma de acceder al mundo, brindan las herramientas para que se rompan las barreras del tiempo y espacio, ofrecen información y conocimiento. Pero, no hay que perder de vista que los medios también pueden servirle a uno para mostrarse al mundo, y no sólo para ver al mundo. Es decir, son una doble vía -así sea desigual- de acceso e interacción social. 
Un resumen de lo que se ha dicho hasta ahora podría sugerir que lo relacionado con la ciudad muestra que «los medios de comunicación constituyen hoy espacios decisivos de la visibilidad y del reconocimiento social». Ello significa que la comunicación (especialmente la mediada) se convierte en espacio de lucha y en lugar de enunciación y negociación de las identidades -locales, minoritarias, periféricas, etc.- que reclaman el «derecho a ser visto, que equivale al derecho de existir/contar socialmente, tanto en el terreno individual como en el colectivo» (MARTÍNBARBERO, 2002: 108-109). Así las cosas, tender los puentes y tejer las redes entre la comunicación y la educación no sólo es estratégico sino necesario. Algunos de estos enlaces ya están dados en las cercanías que brinda la cotidianidad, ahora el reto es explicitarlos y potenciarlos desde una perspectiva académica y formativa.

\section{Proceso metodológico}

Cada sesión de trabajo con los maestros tuvo dos componentes, uno teórico y otro práctico. El componente teórico abordó los aspectos conceptuales más relevantes del ejercicio investigativo. Se buscó problematizar los conceptos y ver cómo éstos se aplicaban en la práctica docente de los maestros o cómo se vivían en su cotidianidad, bien fuera en el marco de las instituciones educativas o en el contexto que estas alcanzan a influir. Es decir, también ha habido interés por contextualizar los aspectos teóricos y tratar de identificar cómo éstos quedan evidentes en prácticas concretas del día a día.

El componente práctico se centró en pequeños relatos biográficos que realizaron los maestros participantes en el proceso de investigación (docentes de educación básica y media vocacional del municipio de Popayán). Estos escritos, elaborados algunos en clave de breves historias de vida, daban cuenta de las experiencias personales de cada asistente en relación con tres espacios urbanos significativos: el barrio, la institución educativa y la ciudad en general. Dichas experiencias se referían, fundamentalmente, a sus años de juventud, de manera tal que se partió de la propia representación que hicieron los maestros de cuando eran jóvenes; entendiendo, en principio, la juventud como una parte del ciclo vital. 
La investigación contempló, además, dos talleres prácticos. El primer taller se desarrolló en dos etapas. La primera buscaba:

a) que se compartieran los relatos biográficos de los participantes, b) que comentaran entre sí los puntos comunes y diferentes de los relatos, y c) que se resaltaran los puntos comunes y se intentara establecer la importancia de ese punto en común.

La segunda etapa del taller consistió en asociar los aspectos más relevantes de los relatos biográficos a alguna -o varias, si era el caso- de las diferentes dinámicas urbanas trabajadas en el componente teóricoconceptual. Este taller se centró fundamentalmente en una dimensión histórica y de evocación, así como en la dinámica urbana de la transitoriedad.

El segundo taller se enfocó en los procesos y medios de comunicación que emplean los maestros en su práctica pedagógica o que están a su alcance en el entorno inmediato. En esta etapa el trabajo giró en torno a la cotidianidad de las prácticas educativas de los maestros y sus instituciones. Se buscaba identificar los espacios o medios de comunicación disponibles e indagar cómo éstos podrían usarse más y mejor para estudiar la ciudad y sus identidades culturales.

El trabajo con los maestros se llevó a cabo en subgrupos que oscilaban entre cinco y ocho personas. Al final de cada taller se hacía una plenaria. Allí cada subgrupo exponía lo que había discutido y entregaba una relatoría con la síntesis y las conclusiones a las que había llegado después de debatir, argumentar y concertar. Dichas relatorías han sido claves para la posterior etapa de sistematización de los resultados de los talleres.

Trabajar a partir de los relatos de los asistentes ha sido muy importante para determinar qué sentidos le otorga cada uno la ciudad, a partir de los espacios vitales que frecuentan o que frecuentaron en su juventud. Se buscó, además, establecer qué sentidos se dan de manera individual y cuáles en forma colectiva. Esta búsqueda por el sentido -que en el marco de la investigación se realizó como grupo- pretendía rastrear la importancia del territorio en relación con los procesos comunicativos que se despliegan en él (¿qué se comunica, cómo se comunica, para qué se hace, quién comunica?). Así, los talleres estuvieron enfocados en encontrar las relaciones que a partir de los medios de comunicación se tejen en función de la visibilidad. 


\section{Sistematización de resultados y hallazgos}

Una vez terminados los talleres se procedió a sistematizar lo consignado por los maestros en las respectivas relatorías. Se buscaron puntos comunes entre unos relatos y otros, y se procuró determinar en qué consistían las principales diferencias. Un primer hallazgo al respecto tiene que ver con la importancia que se le daba al espacio en los relatos biográficos, lo cual significa que la espacialidad se constituye en categoría determinante a la hora de entender las relaciones de los maestros (como sujetos sociales) con la ciudad.

Aquí la espacialidad se configura como la gran dinámica urbana que encierra a otras. Esta espacialidad surge de asumir el espacio como concepto y se establece a partir de las relaciones espacio-sociedad, y lugar vivido-concebido. El lugar, gracias a un «ejercicio» de uso, sentido de pertenencia y apropiación, se convierte en territorio, cuyo sentido se lo dan los habitantes. Por tanto, la espacialidad es una dimensión de la vida social y urbana que conecta la experiencia vital con referentes espaciales; así el espacio termina siendo un producto histórico-social.

\section{El sujeto y su relación con el espacio}

Encontramos entonces que desde la perspectiva biográfica la relación con el espacio - a partir de los usos y apropiaciones de éste, y de lo que éste evoca- es sumamente importante, sobre todo en lo relacionado con procesos de socialización. En esta perspectiva se encontró que el espacio ha sido clave para que se produjeran tres tipos de socialización.

El primer tipo de socialización está relacionado con eventos de la vida cotidiana. Aparecen entonces en los relatos referencias al barrio de la infancia o de la juventud; se nombran con frecuencia lugares de encuentro, las relaciones con los vecinos y espacios en los que se compartía con familiares y amigos. En este tipo de socialización se resaltan algunas actividades concretas y recurrentes realizadas en los barrios; como por ejemplo, los campeonatos deportivos que se organizaban en las calles, las conversaciones interminables en las tiendas y los juegos infantiles de diversa índole que van cambiando con el paso de los años. Otras actividades de socialización relacionadas con el espacio, pero no necesariamente con el barrio, son los tradicionales «paseos de olla», que consistían en paseos familiares o de amigos por algún río o balneario cercano a la ciudad. Lo 
característico de estos paseos es que los asistentes al mismo preparaban el almuerzo en el sitio en donde habían ido a pasear. Finalmente, los relatos que asocian la socialización a partir de lo cotidiano también mencionan los primeros viajes que se realizaron y que generalmente ocurrían en épocas vacacionales.

Recuerdo con mucho cariño los paseos desde la casa paterna a los diferentes ríos de la ciudad, eran deliciosos. Me bañaba en compañía de amigos, esto lo hacíamos los fines de semana. Uno de esos lugares era el río Saté, allí cocinábamos, pescábamos y nos relacionábamos con la naturaleza (Eduardo, profesor de básica primaria).

Un segundo tipo de socialización en relación con el espacio es aquella que se da en los espacios y tiempos de formación (moratoria social $^{1}$ ). Los relatos daban cuenta de vivencias escolares tanto en el colegio como en la universidad. Salían a relucir entonces experiencias asociadas a las clases, las fiestas de integración de compañeros, los encuentros ocasionales que terminaban en programa de ocio, las salidas de campo o eventos académicos a los cuales asistían y que les permitían establecer comparaciones entre su ciudad y aquellos lugares que visitaban. Hubo pocos relatos que mencionaron el primer lugar de trabajo como un espacio importante de socialización.

La vida escolar en el Liceo fue muy agradable. Lo que más me gustaba era la Feria de la Ciencia, ahí realizábamos encuentros de danzas, de canto, exposiciones científicas; éramos reconocidos como uno de lo mejores colegios de la ciudad (Gloria, profesora de bachillerato).

Sólo en el tercer tipo de socialización aparece el imaginario instituido ${ }^{2}$ de la ciudad. Aquí los relatos mencionan los recorridos que se hacían por la ciudad y varios coinciden al calificarla como «linda»o «bella». Aparecen referencias espaciales concretas donde se mencionan calles y parques reconocidos del centro histórico (por ejemplo, el parque Benito Juárez o el Julio Arboleda, o la calle de «El Cacho»), también se nombran lugares icónicos de la ciudad como el parque Caldas, El Morro, el cerro de las Tres Cruces o la torre del Reloj; varios de los templos más reconocidos (la Catedral, San Francisco, Santo Domingo, la Ermita, Belén), y algunos museos (el de Historia Natural, el de Arte Religioso, la Casa Museo Valencia). 
Me acuerdo que en Semana Santa mi papá siempre nos llevaba a ver los templos, los monumentos y los museos del centro, todos me parecían muy bellos [...] Cuando mis hermanas y yo empezamos a salir solas del barrio, empezamos a reconocer también algunas calles del centro y algunos lugares importantes de la ciudad como El Morro y el parque Caldas. Después ya sabíamos cómo se llegaba hasta allá (Martha, profesora de básica primaria).

En suma, a partir de los relatos biográficos de los maestros, vemos cómo la espacialidad se vivencia de lo micro a lo macro, se empieza por reconocer y apropiar el espacio más próximo para luego trascender al más lejano.

Además de la espacialidad, en los relatos también aparecen elementos relacionados con los tránsitos y el desplazamiento en el espacio urbano. Estos elementos los podemos asociar con la dinámica urbana de la transitoriedad trabajada en el componente conceptual de la investigación. En este sentido, los relatos biográficos dan cuenta de dos tipos de tránsitos, aquellos que se dan en la vida cotidiana y los que están atravesados por la práctica formativa.

Los relatos que hacen referencia a desplazamientos en ambientes o prácticas cotidianas mencionan los juegos que se daban en los barrios, especialmente aquellos que implicaban el encuentro con la naturaleza. También aparecen testimonios de viajes con la familia o amigos. Para algunos maestros provenientes de zonas rurales, los primeros viajes a Popayán fueron determinantes y esto lo evidenciaron en sus relatos. El desplazamiento hacia la ciudad o a su interior por medio de recorridos es una práctica frecuente sobre la cual no se reflexiona mucho pues se hace por necesidad. El taller permitió pensar sobre este tipo de tránsitos e identificar las prácticas más frecuentes o las más recordadas.

Cuando terminé mis estudios en el colegio de mi pueblo, me vine para la ciudad. Al principio jugaba mucho basket, para conocer amigos e integrarme. Con ellos caminaba en las noches por las calles de la ciudad [...] cuando estudiaba pensaba en mi familia pero cuando me divertía la nostalgia era menor. Ya después, cuando comencé a trabajar aquí, ya me conocía la ciudad, incluso en esa época caminaba mucho porque me iba a pie de mi casa a la escuela donde empecé a trabajar (Armando, profesor de básica primaria).

Desde esta perspectiva es que aparecen en los relatos aquellos tránsitos asociados a los períodos de formación de los maestros. Se men- 
cionan entonces los recorridos hasta el colegio desde el hogar y viceversa; aparecen referencias de caminatas, de los primeros paseos fuera de la ciudad, de las excursiones de grado y de paseos a ciudades cercanas o a Popayán, en el caso de maestros que provienen de zonas rurales. En una etapa cronológica posterior, también se mencionan en los relatos los recorridos de la casa a la universidad y de la universidad a cualquier otro sitio que resulte en el programa del día a día.

Cuando era niño, mi lugar predilecto era la cuadra en horas nocturnas. Después, cuando me dediqué a estudiar en forma, me dediqué a la psicología, dedicaba ya la mayor parte del tiempo a la lectura, leía de todo. Entonces me la pasaba de la casa de mis padres a la biblioteca de la U. No podría decirse que fuera callejero aunque me la pasaba de arriba para abajo andando por las calles de la ciudad, iba donde algún compañero a estudiar o, muy de vez en cuando, a las fiestas que se programaban en la facultad (Carlos, profesor de bachillerato).

\section{Los medios de comunicación disponibles}

El segundo taller se centró en los medios y procesos de comunicación que los maestros tienen a su alcance o gestionan como herramienta útil y proactiva para su práctica pedagógica. Es evidente que el componente de la cotidianidad sobresalió en primera instancia pero también emergieron «nuevos» medios o medios «alternativos» de comunicación, así como la posibilidad de potenciar nuevas instancias o espacios para la comunicación. Recordemos aquí que existe una relación cercana entre el uso y el consumo de medios con la dinámica urbana de la visibilidad.

Con respecto a los medios más usados en la práctica de los maestros, se mencionan desde los convencionales hasta los mediados por una técnica, estos últimos ya fruto de las tecnologías de la información y la comunicación. Las experiencias con éstos son variadas y múltiples y dependen tanto de la edad de cada maestro como de la institución donde éste trabaje.

Los medios de comunicación que más se emplean son los más convencionales, como el periódico mural, la cartelera escolar y los volantes informativos. En un segundo grupo encontramos una visión más institucional del medio y es cuando aparecen los boletines o los periódicos escolares o institucionales; en el caso de las zonas rurales -e incluso en la ciudad, en escuelas y colegios que tienen instalado el sistema- es muy 
común el uso del parlante como un medio de comunicación. Menos usados pero cada vez más frecuentes son los medios de comunicación escolares diseñados para este fin: aquí aparecen las emisoras escolares (o estudiantiles, pues son los alumnos quienes las mantienen con vida) o, incluso, el uso de Internet a través de las páginas web o blogs de colegios. Estos últimos medios todavía son escasos y fundamentalmente urbanos pero se encuentran en constante crecimiento.

Adicionalmente a los medios de comunicación «convencionales», aparecen una serie de espacios de comunicación que se relacionan más con la práctica pedagógica cotidiana o la vida diaria de los maestros y sus instituciones educativas. Estos espacios de comunicación, que algunos efectivamente los usan o que podrían usarse, pueden dividirse entre endógenos y exógenos, según cual sea su relación con las instituciones.

Entre los endógenos encontramos la puerta de entrada al colegio o la escuela, los salones y sus espacios de atención permanente (como los tableros), la sala de profesores, la biblioteca, los lugares de alimentación (restaurante escolar, cafetín, tienda), los lugares que congregan a gran cantidad de personas (aula múltiple, auditorio).

Entre los espacios exógenos que pueden emplearse más y mejor para difundir información, los maestros identificaron: los vehículos de transporte (urbano y escolar), los sitios de encuentro y recreación (el polideportivo y/o el parque del barrio), los sitios de reunión como el salón comunal, las instituciones reconocidas del entorno (bancos, iglesias, almacenes, hoteles, restaurantes, etc.).

Finalmente, los maestros identificaron instancias en las cuales se pueden generar procesos de comunicación ya que su función habitual suele ser el suministro de información. Estas instancias son permanentes y tienen que ver también con la cotidianidad de la vida escolar de los maestros y sus instituciones educativas. Las instancias más comunes son las asambleas o reuniones de padres de familia (también se incluyen aquí las escuelas de padres que existen en algunos colegios y escuelas), y los eventos académicos, deportivos y culturales que se programan a lo largo del año lectivo.

Después de este ejercicio, relacionado con los medios, espacios e instancias de comunicación al alcance de los maestros, es importante proyectar cómo éstos se pueden usar más y mejor para educar sobre la ciudad. En este sentido, no basta con que los medios existan y se emple- 
en, hay que tener claridad acerca de la intención comunicativa, en lo que se refiere a su uso.

De acuerdo con los maestros, la intención comunicativa con que se usan o se pueden usar los medios, espacios e instancias de comunicación que están a su alcance, debe pensarse en función de:

- Suministro de información (variada) sobre: horarios, capacitaciones, cronograma de actividades.

- Promoción de actividades, eventos y tareas pedagógicas

- Sensibilizar sobre temáticas puntuales a públicos específicos (padres de familia, estudiantes, comunidad educativa).

Asimismo, según los maestros, es clave que los medios, espacios e instancias puedan:

- Tener seguimiento, control y evaluación

- Divulgar información actualizada veraz y oportunamente

- Difundir temas de interés comunitario que inviten a la reflexión y al desarrollo de valores.

- Responder a las necesidades de la comunidad, ser llamativos y estar bien ubicados para facilitar su consumo.

- Tener claro el objetivo de la información que se ofrece. Ésta debe ser oportuna, asertiva, participativa y creativa.

En la medida en que la información fluya y circule, es más probable que ésta sea consumida; es decir, la circulación efectiva de la información facilita el consumo de la misma y de alguna manera favorece que a partir de ahí se generen procesos de comunicación más activos e incluyentes. Si en esta perspectiva se incluye el tema de los estudios urbanos o el conocimiento sobre la ciudad que se habita, seguramente la concepción que se tiene de la ciudad estará menos relacionada a un contenido programático y más a su lado vivencial.

\section{Conclusiones}

El trabajo desarrollado con los maestros en la investigación puntual sobre imaginarios de ciudad y comunicación permite concluir lo siguiente: 
La transitoriedad por sí misma no existe en la ciudad, ésta se produce en función del espacio y de la vida cotidiana de cada individuo o de cada grupo que se apropia de los espacios que le son habituales y los convierte en su territorio. Se pasa, entonces, del concepto de ciudad al de prácticas urbanas (DE CERTEAU, 2008), donde, más que racionalizar sobre lo que es, lo que representa o lo que significa la ciudad, se la vive en la cotidianidad de sus ritmos y rutinas.

En este caso, la práctica de la transitoriedad, como dinámica urbana, termina siendo fundamental para comprender la relación entre los sujetos y el espacio. Lo que subyace es el uso y el sentido que se le da al espacio urbano, de forma tal que transitoriedad y espacialidad son claves para que puedan entenderse los procesos de socialización que se producen en relación con el territorio.

En el trabajo con los maestros pudo determinarse que hay tres tipos de socialización: a) la cotidiana, b) la que se da en espacios y tiempos de formación, y c) la que tiene que ver con el imaginario instituido de ciudad. Esta socialización diversa evidencia que la ciudad no se concibe solamente «como un espacio físico sino como un espacio simbólico del desarrollo de la ciudadanía», y por tanto, representa un triple desafío en lo personal y en lo docente: desde los planos político, social y específicamente urbano (ALDEROQUI, 2002: 231-232).

En el caso estudiado y presentado en este trabajo, es fuerte la relación entre juventud y moratoria social, no en vano, parte del imaginario social instituido de Popayán asocia la ciudad con la educación. Un ejemplo concreto de esto lo podemos ver cuando se ha dado por denominar a Popayán como "ciudad universitaria". Esta concepción, que en ocasiones se asume como "slogan" de la ciudad, refuerza la idea de que aquí los jóvenes tienen un tiempo y espacio propicios para su formación académica y cultural, previos a su integración social y laboral, propios del mundo adulto.

Se trata entonces de una suerte de discurso legitimado sobre la ciudad que se logra instalar en el imaginario colectivo. Tanto en el discurso cotidiano sobre la ciudad como en los procesos sociales que lo respaldan, «el discurso sobre la ciudad [...], se habla de la ciudad en términos referenciales, es decir, no se trata de la ciudad que (se) enuncia sino de la que se habla. Este discurso habla "por" y "sobre" la ciudad» (BUENDÍA, 2008: 262). 
En relación con la dinámica urbana de la visibilidad, los maestros identificaron los procesos de comunicación como determinantes para que dicha visibilidad ocurra o no. En este sentido, estos procesos se llevan a cabo gracias a que existen medios, espacios e instancias de comunicación que son utilizados por los maestros.

Para los maestros, es muy importante el uso responsable que se haga de los medios, espacios e instancias que generen procesos de comunicación, pues en la práctica urbana y docente, subyace la idea de «comprender a la ciudad como un escenario o lugar de la experiencia y de las prácticas sociales y culturales» (PEREIRA, 2007: 91).

No obstante, vale la pena resaltar que si bien hay un interés comunicativo por parte de los maestros, aún prima una visión instrumental de la comunicación, donde el peso recae de manera especial en el uso y la utilidad de la información que se divulgue por los medios. Pese a esto, la comunicación, según los maestros, es clave para su práctica educativa y desde ella es más fácil comprender el complejo entramado de las dinámicas urbanas. 


\section{Referencias}

ALDEROQUI, S. (2002). «Para qué enseñar la ciudad?», en S. ALDEROQUI \& P. PENCHANSKY (comps.) Ciudad y ciudadanos. Aportes para la enseñanza del mundo urbano. 67-93. Buenos Aires, Piados.

BUENDÍA, A. (2008). «Ciudad, espacio público y comunicación: Una reflexión en torno al discurso pedagógico de y sobre la ciudad», en: M. CÓRDOVA (coord.) Lo urbano en su complejidad: una lectura desde América Latina. 257-266. Quito, FLACSO.

BUENDÍA, A. (2006). Jóvenes, radio y ciudadanía. Popayán, Axis Mundi.

BUENDÍA, A. \& HURTADO, D. (2009). Imaginarios del espacio público en jóvenes de Popayán. Proyecto de investigación. Popayán, Universidad del Cauca.

CARRIÓN, F. \& WOLLRAD, D. (comps.). (1999). La ciudad, escenario de comunicación. Quito, Flacso.

DE CERTEAU, M. (2008). «Andar la ciudad», en Bifurcaciones [online]. núm. 7, URL: $<$ www.bifurcaciones.cl/007/reserva.htm>.

FEIXA, C. (1998). «La ciudad invisible. Territorios de las culturas juveniles», en H. CUBIDES, M. C. LAVERDE \& C. E. VALDERRAMA (eds.), "Viviendo a toda". Jóvenes, territorios culturales y nuevas sensibilidades. 83-109. Santafé de Bogotá D.C, Universidad Central - DIUC - Siglo de Hombre.

HURTADO, D. (2004). «Reflexiones sobre la teoría de imaginarios: una posibilidad de comprensión desde lo instituido y la imaginación radical», en Cinta de Moebio n. 21. Santiago, Universidad de Chile.

MARTÍN-BARBERO, J. (2002). La educación desde la comunicación. Buenos Aires, Norma. MARTÍN-BARBERO, J. (1995). Pre-textos. Conversaciones sobre la comunicación y sus contextos. Cali, Universidad del Valle.

PEREIRA, J. M. (2007). «Ciudad, comunicación y construcción de lo público», en C. M. Yori (Ed.) Espacio público y formación ciudadana. 75-94. Bogotá, PUJ.

PÉREZ TORNERO, J. M. (1998). «El ansia de identidad juvenil y la educación. Del narcisismo mediático contemporáneo y las estrategias educativas», en H. CUBIDES, M. C. LAVERDE \& C. E. VALDERRAMA (eds.), "Viviendo a toda". Jóvenes, territorios culturales y nuevas sensibilidades. 263-277. Santafé de Bogotá D.C., Universidad Central - DIUC Siglo de Hombre.

ULLOA, A. (2000). Globalización, ciudad y representaciones sociales. El caso de Cali. Medellín, UPB. 


\section{Notas}

1 Este concepto está asociado a la idea de juventud como etapa de transición y, sobre todo, de formación. Dicha idea se sustenta en un imaginario social instituido que señala que es en la juventud cuando los sujetos se preparan (incluso académicamente) para la integración social. Así, la gente joven es quien debe formarse con valores, destrezas y habilidades para formar parte, después, del mundo adulto. (HURTADO, 2004).

2 El imaginario social instituido se asume como una matriz de sentido existencial que, desde lo subjetivo, otorga relevancia al discurso, al pensamiento y a la acción social. Para Herrera (2004), los imaginarios instituidos son aquellos esquemas de representación que se construyen socialmente y logran cierta legitimidad y hegemonía, de forma tal que a partir de ellos se puede dar cuenta de una realidad social determinada.

Recebido: 16/06/2009

Aprovado: 06/04/2010

Contato:

Universidad del Cauca

Facultad de Ciencias Naturales, Exactas y de la Educación

Cra 2\# 3N-111

Tulcán - Popayán

Cauca

Colombia 